\title{
Tratamento cirúrgico de emergência no acidente vascular cerebral isquêmico. Afinal, o que há de evidências?
}

\author{
Robson Luis Oliveira de Amorim', Wellingson Silva Paiva', Eberval Gadelha
}

Figueiredo $^{2}$, Almir Ferreira de Andrade ${ }^{3}$, Manoel Jacobsen Teixeira ${ }^{4}$

Divisão de Neurocirurgia do Hospital das Clínicas da Universidade de São Paulo (HC-FMUSP), SP, Brasil

\section{RESUMO}

Contexto: O manuseio dos pacientes com infarto cerebral isquêmico inclui várias abordagens de tratamento. O tratamento cirúrgico é raramente necessário, entretanto os médicos gerais, neurologistas e neurocirurgiões devem estar atentos para essa possibilidade. Objetivo: Esta revisão visa sistematizar o tratamento cirúrgico com base em evidências nos acidentes vasculares isquêmicos. Conclusões: Apesar da crescente pesquisa envolvendo os acidentes vasculares, a taxa de mortalidade após a tentativa de tratamento cirúrgico pouco se tem alterado nas últimas décadas. Questões como quando ou a quem indicar uma craniectomia descompressiva no infarto isquêmico hemisférico são motivos de controvérsias no âmbito neurocirúrgico, e os dados da literatura são pouco esclarecedores.

\section{PALAVRAS-CHAVE}

Acidente cerebral vascular, cirurgia. Infarto cerebral, cirurgia.

\section{ABSTRACT}

Surgical treatment of acute cerebral infarction. What are the evidences?

Background: Management of patients with acute ischemic stroke remains multifaceted and includes several aspects. The surgical treatment is rarelly necessary but general physicians, neurologists and neurosurgeons must be aware of that possibility. Objective: This review intends to systematize the surgical treatment based on evidences in ischemic strokes. Conclusions: In spite of crescent researches involving the cerebro vascular strokes, the mortality after surgical treatment did not decrease significantly in last decades. When or in whom a decompressive craniotomy should be done in hemispheric infarct are questions that lead to fervorous discussions in the neurosurgical context, and, unfortunatelly, the literature is still controversial. Because many of the recommendations are based on limited data, additional research on treatment of acute ischemic stroke is needed.

\section{KEY WORDS}

Stroke, surgery. Cerebral infarction, surgery.

\section{Introdução}

O manuseio de pacientes com acidente vascular cerebral isquêmico mantém-se multifacetado e inclui uma série de cuidados, mas muitos aspectos ainda não foram adequadamente testados em ensaios clínicos controlados. Os trombolíticos endovenosos são a chave do tratamento, porém, quando se perde a janela terapêutica, quando o infarto é extenso ou localizado no cerebelo, o tratamento cirúrgico pode ser uma opção. Este artigo abordará as principais evidências no tocante ao tratamento cirúrgico de emergência para pacientes com infarto maligno da artéria cerebral média e com infarto cerebelar.

1 Médico-assistente. Divisão de Neurocirurgia do Hospital das Clínicas da Faculdade de Medicina da Universidade de São Paulo (HC-FMUSP). 2 Médico-supervisor. Divisão de Neurocirurgia do HC-FMUSP.

3 Coordenador do Pronto-Socorro de Neurocirurgia do HC-FMUSP.

4 Professor Titular de Neurocirurgia do Departamento de Neurologia da FMUSP. 


\section{Infarto maligno em território de artéria cerebral média}

Pode-se predizer, quando pacientes se apresentam com déficits intensos como hemiplegia, afasia, desvio forçado do olho e da cabeça, associados a alterações tomográficas precoces $(<12 \mathrm{~h})$ como hipoatenuação extensa maior que $50 \%$ do território da artéria cerebral média (ACM) e apagamento de sulcos, que a evolução será maligna, fato que ocorre em cerca de $10 \%$ de todos os acidentes vasculares encefálicos, ${ }^{11,17}$ com mortalidade de até $80 \%$ (Quadro 1). ${ }^{11}$ Von Kummer e cols. ${ }^{24}$ demonstraram que hipodensidade comprometendo área maior que $50 \%$ do território da ACM tem uma especificidade de $94 \%$ e sensibilidade de $61 \%$ para infarto maligno. Geralmente, esses pacientes apresentam rebaixamento do nível de consciência durante as primeiras 48 horas e podem evoluir para óbito em até 96 horas, com herniação transtentorial. Apesar do tratamento clínico otimizado, com o uso de manitol, indução de coma barbitúrico, hiperventilação e hipotermia, a mortalidade pode chegar a $80 \%{ }^{11}$

Considerando a alta mortalidade e o manejo clínico insatisfatório nessas situações, muitos autores têm defendido conduta cirúrgica agressiva como a hemicraniectomia para o tratamento de infarto hemisférico (Figura 1). A craniectomia descompressiva tem sido

\section{Quadro 1}

Fatores preditivos de evolução para infarto maligno da ACM

Achados tomográficos precoces $(<12 \mathrm{~h})$ como hipoatenuação extensa (maior que $50 \%$ do território da $\mathrm{ACM}$ ) e apagamento de sulcos ${ }^{15}$

Acometimento de outros territórios vasculares ${ }^{13}$

Náuseas e vômitos precoces

Escala do NIH $\geq 20$ para infarto em hemisfério

esquerdo $\mathrm{e} \geq 15$ para infarto à direita

Hipertensão, insuficiência cardíaca, leucocitose $\mathrm{e}^{15}$

Aumento precoce da proteína $\mathrm{S} 100 \mathrm{~B}^{7}$ usada para a redução da pressão intracraniana (PIC) em diversas situações. Em 1905, Cushing ${ }^{5}$ descreveu esse procedimento para alívio da pressão intracraniana em pacientes com tumores intracranianos. Desde essa época, já se descreveu essa cirurgia para o tratamento de traumatismo cranioencefálico, hematoma subdural, vasoespasmo, encefalite, hematoma intracraniano, trombose venosa cerebral e infarto cerebelar. Em 1968, Greenwood $^{9}$ fez uso desse procedimento em infartos supratentoriais, reduzindo a mortalidade para menos de $50 \%$. A técnica utilizada era hemicraniectomia descompressiva e ressecção do parênquima necrosado. Em 1971, foi tentada craniectomia bifrontal sem sucesso, e somente em 1981 Rengachary e cols. ${ }^{18}$ descreveram os primeiros casos de craniectomia sem remoção de tecido cerebral necrosado. A partir disso, muitos autores têm demonstrado o benefício dessa conduta em pacientes com infarto hemisférico.

Rieke e cols., ${ }^{19} \mathrm{em} 1995$, em um estudo prospectivo e controlado não randomizado, avaliaram 53 pacientes; em 32 deles foi realizado tratamento cirúrgico descompressivo. A mortalidade no grupo cirúrgico foi de 34\% e no grupo tratado clinicamente, de $76 \%$. Yang e cols. ${ }^{28}$ obtiveram redução da mortalidade para $10 \%$ no grupo tratado cirurgicamente. Outros estudos também chegaram a resultados semelhantes, em que foi encontrada grande redução da mortalidade.

Qual seria o melhor momento para a realização da craniectomia descompressiva? Muitos autores recomendam craniectomia precoce, isto é, antes de o paciente apresentar sinais de deterioração clínica. ${ }^{6,8,16}$ Em um estudo prospectivo ${ }^{21}$ em que foram avaliados 63 pacientes com acidente vascular isquêmico extenso de ACM, a hemicraniectomia precoce (média de 21 horas) levou a uma diminuição de mortalidade de $34 \%$ para $16 \%$. O prognóstico funcional nesses pacientes, de acordo com o índice de Barthel, teve uma média de 70 em comparação com 62,6 no grupo operado tardiamente. Entretanto, não houve diferença estatis-

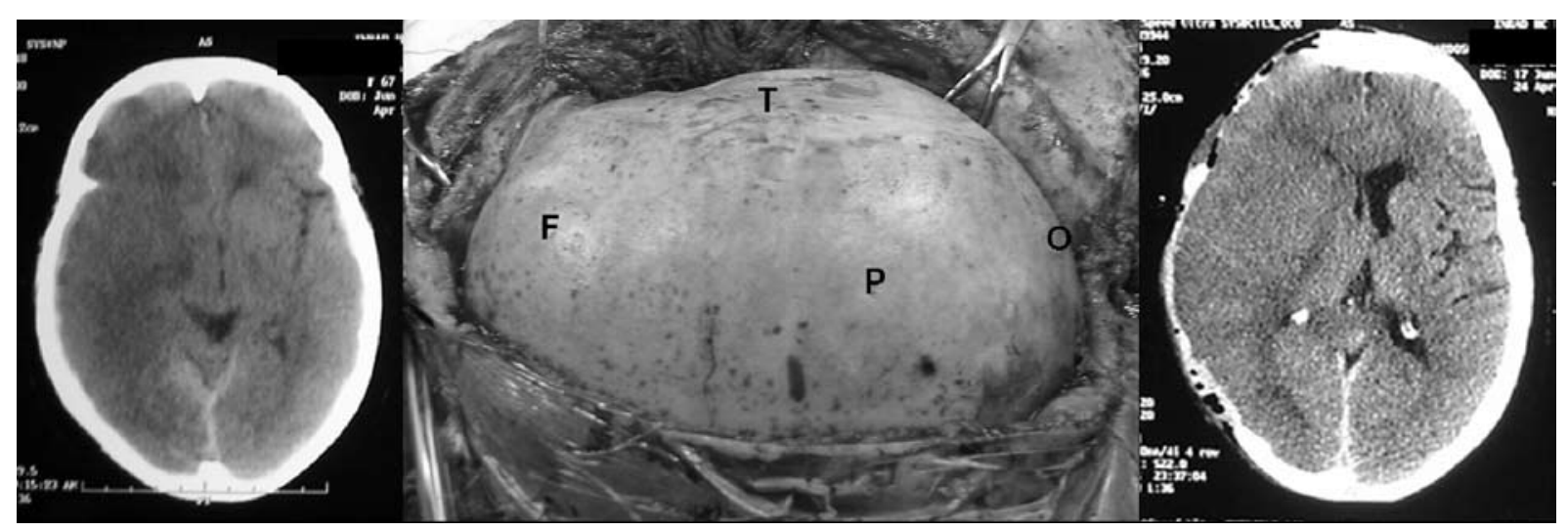

Figura 1-Esquerda: acidente vascular cerebral isquêmico hemisférico. Centro: craniotomia ampla seguida de duroplastia. Direita: controle pós-operatório. 
ticamente significante nos dois grupos, apesar de no grupo dos operados precocemente todos serem capazes de caminhar curtas distâncias sem apoio e de se integrar adequadamente com familiares e no ambiente social, segundo relatos.

Em outro estudo controlado ${ }^{2}$ foi evidenciada redução de $36,7 \%$ para $8,7 \%$ na mortalidade relativa a pacientes - cujo diagnóstico de infarto maligno foi determinado com imagens de perfusão - que foram operados com menos de 6 horas de evolução. Postula-se que a espera por sinais de herniação pode piorar o prognóstico em razão da lesão mesencefálica irreversível, ${ }^{21}$ o que é perfeitamente compreensível em um paciente jovem, com menor atrofia cerebral, que pode não tolerar edemas cerebrais maciços.

De acordo com a literatura recente, aqueles que provavelmente mais se beneficiariam com o procedimento cirúrgico seriam os pacientes jovens, ${ }^{2,19,26}$ principalmente aqueles com menos de 50 anos. Yao e cols., ${ }^{29}$ em estudo retrospectivo com 25 pacientes operados, obtiveram mortalidade de $7,7 \%$ em menores de 60 anos e de 33,3\% em maiores de 60 anos. No primeiro grupo, o índice de Barthel teve uma média de 75,42 , enquanto no segundo grupo nenhum paciente obteve índice maior que 60. Entretanto, a idade de corte para a decisão da realização da hemicraniectomia baseia-se prioritariamente no bom senso. Um paciente de 75 anos, hígido, sem comorbidades, provavelmente se beneficiaria mais de um procedimento cirúrgico do que um de 50 anos, com hipertensão e insuficiência cardíaca. Em um país como o nosso, em que a expectativa de vida média é de 71,3 anos, 65 seria uma idade de corte aceitável.

A principal controvérsia sobre essa medida cirúrgica heroica seria a qualidade de vida que esses pacientes teriam por conta da afasia em caso de infartos em hemisfério dominante. Via de regra, pesquisadores do passado não realizavam a craniectomia quando o hemisfério dominante era o comprometido. Em uma revisão crítica de 138 pacientes, ${ }^{10}$ o prognóstico funcional de 27 pacientes que foram submetidos à cirurgia descompressiva à esquerda foi semelhante ao de outros 111 pacientes submetidos à descompressão do lado direito, sugerindo que o lado do infarto não deve ser um critério para definição da conduta cirúrgica, ${ }^{22}$ especialmente se esses pacientes estiverem com alguma função de linguagem na admissão. Recentemente, em uma análise de três estudos clínicos randomizados (o francês DECIMAL, o holandês HAMLET e o alemão DESTINY), verificou-se mortalidade de $22 \%$ dos pacientes em que a craniectomia descompressiva foi realizada nas primeiras 48 horas e de $71 \%$ no grupo que não passou por procedimento cirúrgico. O número de pacientes com prognóstico funcional favorável (escala de Rankin modificada $\leq 3$ ) dobrou em relação aos pacientes não operados. Os estudos DECIMAL e DESTINY não foram finalizados em razão da diferença significante da mortalidade entre o grupos cirúrgicos e não cirúrgicos, em favor do primeiro. Nesses estudos, os pacientes com mais de 60 anos foram excluídos. ${ }^{23}$

\section{O papel da monitorização da pressão intracraniana}

Alguns autores ${ }^{25,26,30}$ sugerem a monitorização da PIC para decisão da intervenção terapêutica. Foi determinado que pacientes com PIC elevada tendem a ter pior prognóstico..$^{20,21}$ Entretanto, o valor inicial da PIC não permite informação precoce da evolução clínica. ${ }^{20}$ Acreditamos, portanto, que o quadro clínicoradiológico é suficiente para determinar o momento da hemicraniectomia.

\section{O procedimento cirúrgico}

A hemicraniectomia para infartos supratentoriais necessita o máximo de ressecção de tecido ósseo, como demonstrado por Kerr ${ }^{14}$ em casos de inchaço cerebral pós-traumático e constatado por Rieke e cols., ${ }^{19}$ que observaram herniação transcalvariana quando a ressecção óssea foi incompleta. A retirada do retalho ósseo determina uma redução da PIC em $15 \% .{ }^{12}$ Realiza-se uma hemicraniectomia, incisão da dura-máter em arco ou em cruz e expansão com fáscia do músculo temporal, pericrânio ou substituto biossintético dural (Figura 1). Segundo Jourdan e cols., ${ }^{12}$ a duroplastia pode reduzir a PIC em até $70 \%$. Woertgen e cols. ${ }^{27}$ relataram aumento da mortalidade quando não se realizava a duroplastia $(58 \%$ versus $14 \%, \mathrm{p}<000,5)$. A dura-máter é ancorada para prevenir hematoma extradural. O músculo temporal é suturado, seguindo-se o fechamento da pele. O retalho ósseo pode ser preservado em um banco de ossos ou no tecido subcutâneo abdominal. ${ }^{2}$ Quando o paciente apresenta recuperação funcional, procede-se à cranioplastia, geralmente após 4 a 12 semanas.

\section{Aspecto funcional da cirurgia}

Problemas psicológicos frequentemente envolvem o paciente e a família. Foi válida a realização de um procedimento tão invasivo? Em um estudo recente, em que foram avaliados 48 pacientes retrospectivamente, não houve diferença estatística no índice de qualidade de vida dos pacientes operados à esquerda ou à direita nem daqueles com ou sem afasia e $83 \%$ dos pacientes sobreviventes e familiares ficaram satisfeitos com o procedimento. ${ }^{27} \mathrm{Como} \mathrm{já} \mathrm{descrito,} \mathrm{o} \mathrm{principal} \mathrm{fator} \mathrm{prog-}$ nóstico é a idade; resultado pior (índice de Barthel $<50$ ) 


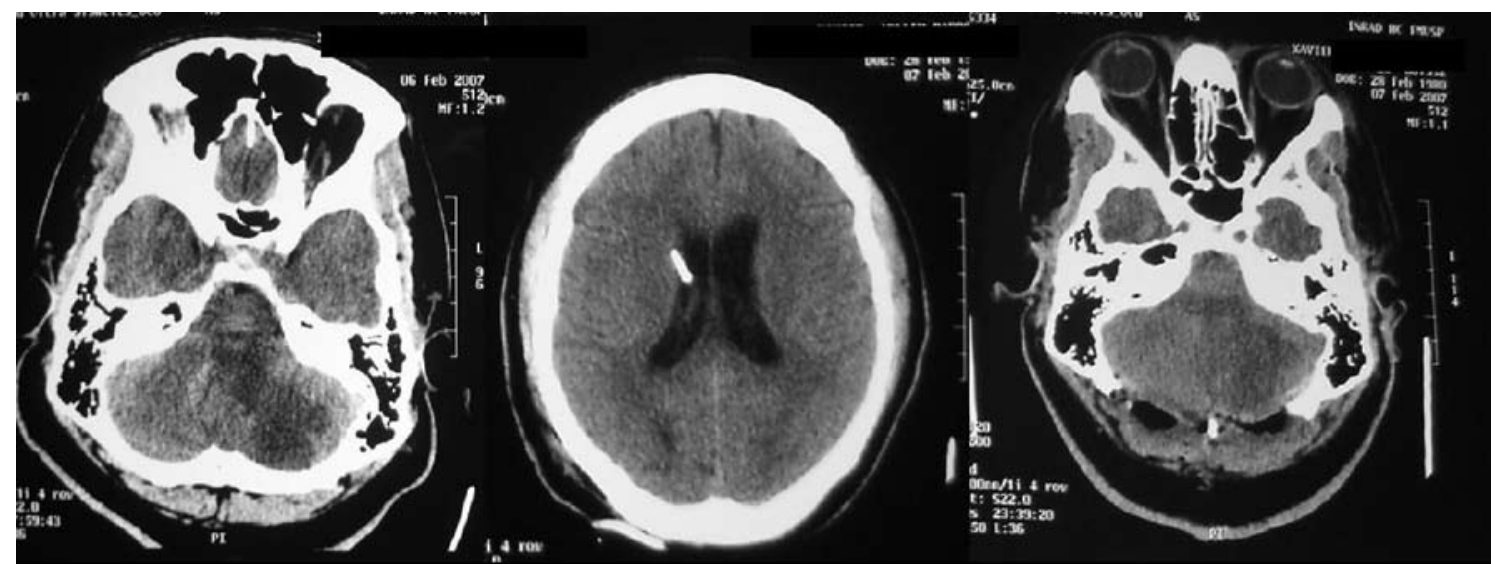

Figura 2 - Paciente masculino, 27 anos, cardiopata. Apresentou quadro súbito de ataxia de marcha, náuseas e vômitos. No segundo dia pós-icto evoluiu com sonolência. Esquerda: TC de crânio revelando acidente vascular isquêmico em hemisfério cerebelar esquerdo. Centro: realizada derivação ventricular externa. Direita: craniotomia suboccipital ampla com duroplastia.

é observado nos pacientes com déficits neurológicos severos na admissão, duração de tratamento intensivo, necessidade de ventilação mecânica ${ }^{7}$ e tratamento cirúrgico tardio. ${ }^{16}$ Avaliações mais direcionadas e precisas são ainda necessárias para a resolução de uma dúvida no mínimo intrigante. No momento, com os estudos disponíveis podemos seguir em uma direção. Em um futuro breve saberemos se esse foi o melhor caminho.

\section{Infarto cerebelar}

Pacientes com infarto cerebelar extenso podem evoluir rapidamente para coma profundo por causa da compressão do tronco encefálico ou hidrocefalia. Nesses casos, o tratamento cirúrgico precoce é imperativo, com a realização de derivação ventricular externa seguida de craniectomia suboccipital com abertura do forame magno (Figura 2). ${ }^{3}$ Esse tratamento, no caso de infartos cerebelares extensos, é considerado classe I com nível B de evidência pela American Heart Association e pela American Stroke Association. ${ }^{1}$ A derivação ventricular externa isolada deve ser evitada pelo risco de herniação cerebelar ascendente e por não evitar a compressão do tronco.

\section{Conclusões}

Apesar da controvérsia ainda existente na literatura relativa à craniectomia no infarto hemisférico, há uma tendência a se adotar um tratamento agressivo e precoce em pacientes selecionados. Mais estudos controlados e randomizados são necessários para nos orientar quanto ao melhor momento de se indicar a cirurgia, quais exa- mes podem predizer com alto grau de confiabilidade os pacientes que podem apresentar uma evolução desfavorável e qual a melhor técnica cirúrgica.

Com relação ao infarto cerebelar, observa-se uma homogeneidade no padrão de dados, o que, necessariamente, não invalida novas pesquisas. Estudos sobre qualidade de vida são escassos e fundamentais para formulação de ideias em busca de alternativas cada vez melhores para os nossos pacientes.

\section{Referências}

1. Adams HP Jr, Del Zoppo G, Alberts MJ, Bhatt DL, Brass L, Furlan A, et al. American Heart Association, American Stroke Association Stroke Council, Clinical Cardiology Council, Cardiovascular Radiology and Intervention Council, and the Atherosclerotic Peripheral Vascular Disease and Quality of Care Outcomes in Research Interdisciplinary Working Groups. Guidelines for the early management of adults with ischemic stroke. Stroke. 2007;38:1655-711.

2. Carter BS, Ogilvy CS, Candia GJ, Rosas HD, Buonanno F. One-year outcome after decompressive surgery for massive nondominant hemispheric infarction. Neurosurgery. 1997;40:1168-76.

3. Chen HJ, Lee TC, Wei CP. Treatment of cerebellar infarction by decompressive suboccipital craniectomy. Stroke. 1992;23:957-61.

4. Cho DY, Chen TC, Lee HC. Ultra-early decompressive craniectomy for malignant middle cerebral artery infarction. Surg Neurol. 2003;60:227-32.

5. Cushing $\mathrm{H}$. The establishment of cerebral hernia as a decompressive measure of inaccessible brain tumors; with the description of intermuscular methods of making the bone defect in temporal and occipital regions. Surg Gynecol Obstet. 1905;1:297-314.

6. Doerfler A, Forsting M, Reith W, Staff C, Heiland S, Schäbitz WR, et al. Decompressive craniectomy in a rat model of "malignant" cerebral hemispherical stroke: experimental support for an aggressive therapeutic approach. J Neurosurg. 1996;85:853-9. 
7. Foerch C, Lang JM, Krause J, Raabe A, Sitzer M, Seifert V, et al. Functional impairment, disability, and quality of life outcome after decompressive hemicraniectomy in malignant middle cerebral artery infarction. J Neurosurg. 2004; 101:248-54.

8. Forsting M, Reith W, Schäbitz WR, Heiland S, Von Kummer R, Hacke W, et al. Decompressive craniectomy for cerebral infarction: an experimental study in rats. Stroke. 1995;26:259-64.

9. Greenwood Jr J. Acute brain infarctions with high intracranial pressure: surgical indications. Johns Hopkins Med J. 1968;122:254-60.

10. Gupta RE, Connolly S, et al. Hemicraniectomy for massive middle cerebral artery territory infarction: A systematic review. Stroke. 2004;35:539-43.

11. Hacke W, Schwab S, Horn M, Spranger M, De Georgia M, Von Kummer V. "Malignant" middle cerebral artery territory infarction. Arch Neurol. 1996;53:309-15.

12. Jourdan C, Convert J, Mottolese C, et al. Evaluation of the clinical benefit of decompression hemicraniectomy in intracranial hypertension not controlled by medical treatment. Neurochirurgie. 1993;39:304-10.

13. Kasner SE, Demchuk AM, Berrouschot J, Schmutzhard E, Harms L, Verro P, et al. Predictors of fatal brain edema in massive hemispheric ischemic stroke. Stroke. 2001;32:2117-23.

14. Kerr FW. Radical decompression and dural grafting in severe cerebral edema. Mayo Clin Proc. 1968;43:852-64

15. Krieger DW, Demchuk AM, Kasner SE, Jauss M, Hantson L. Early clinical and radiological predictors of fatal brain swelling in ischemic stroke. Stroke. 1999;30:287-92.

16. Mori K, Nakao Y, Yamamoto T, Maeda M. Early external decompressive craniectomy with duroplasty improves functional recovery in patients with massive hemispheric embolic infarction: timing and indication of decompressive surgery for malignant cerebral infarction. Surg Neurol. 2004;62:420-9.

17. Moulin DE, Lo R, Chiang J, Barnett HJM. Prognosis in middle cerebral artery occlusion. Stroke. 1985;16:282-4.

18. Rengachary SS, Batnitzky S, Moranz RA. Hemicraniectomy for acute massive cerebral infarction. Neurosurgery. 1981;8:321-8.

19. Rieke K, Schwab S, Krieger D. Decompressive surgery in space-occupying hemispheric infarction: results of an open prospective trial. Crit Care Med. 1995;23:1576-8.

20. Schwab S, Aschoff A, Spranger, et al. The value of ICP monitoring in acute hemispheric stroke. Neurology. 1996;47:393-8.
21. Schwab S, Steiner T, Aschoff A, Schwarz S, Steiner $\mathrm{HH}$, Jansen $\mathrm{O}$, et al. Early hemicraniectomy in patients with complete middle cerebral artery infarction. Stroke. 1998;29:1888-93.

22. Solomon NA, Glick HA, Russo CJ, Lee J, Schulman KA. Patient preferences for stroke outcomes. Stroke. 1994; 25:1721-5.

23. Vahedi K, Hofmeijer J, Juettler E, Vicaut E, George B, Algra $A$, et al. Early decompressive surgery in malignant infarction of the middle cerebral artery: a pooled analysis of three randomised controlled trials. Lancet Neurol. 2007;6:215-22.

24. Von Kummer R, Meyding-Lamade U, Forsting M, Rosin L, Rieke K, Sartor K, et al. Sensitivity and prognostic value of early computed tomography in middle cerebral artery trunk occlusion. AJNR. 1994;15:9-15.

25. Wijdicks EFM, Diringer MN. Middle cerebral artery territory infarction and early brain swelling: progression and effect of age on outcome. Mayo Clin Proc. 1998;73:829-36.

26. Wijdicks EFM, Schievink WI, McGough PF. Dramatic reversal of the uncal syndrome and brain edema from infarction in the middle cerebral artery territory. Cerebrovasc Dis. 1997;7:349-52.

27. Woertgen C, Erban P, Rothoerl RD, Bein T, Horn M, Brawanski A. Quality of life after decompressive craniectomy in patients suffering from supratentorial brain ischemia. Acta Neurochir (Wien). 2004;146:691-5.

28. Yang XF, Yao Y, Hu WW, Li G, Xu JF, Zhao XQ, et al. Is decompressive craniectomy for malignant middle cerebral artery infarction of any worth? J Zhejiang Univ Sci. 2005;B6:644-9.

29. Yao Y, Liu W, Yang X, Hu W, Li G. Is decompressive craniectomy for malignant middle cerebral artery territory infarction of any benefit for elderly patients? Surg Neurol. 2005;64:165-9.

30. Young PH, Smith KR, Dunn RC. Surgical decompression after cerebral hemispheric stroke: indications and patient selection. South Med J. 1982;75:473-4.

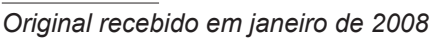

Aceito para publicação em dezembro de 2008

\section{Endereço para correspondência}

Robson Amorim

Rua Oscar Freire, 1811, ap. 12

05409-011 - São Paulo, SP, Brasil

E-mail:amorim.robson@uol.com.br 